\title{
Oral représenté et diachronie : étude des incises en français médiéval
}

\author{
Guillot, Céline \\ Icar (CNRS - Lyon2 / ENS), IUF \\ celine.guillot@ens-lyon.fr \\ Prévost, Sophie \\ Lattice (CNRS - ENS / Sorbonne Nouvelle Paris3) \\ sophie.prevost@ens.fr \\ Lavrentiev, Alexei \\ Icar (CNRS - Lyon2 / ENS) \\ alexei.lavrentev@ens-lyon.fr
}

\section{Introduction}

L'étude des marques d'oralité présentes dans les textes écrits en français médiéval (voir notamment les recherches sur les mots du discours, les adverbes énonciatifs, les termes d'adresse, les interjections, etc. ${ }^{1}$ ) et les recherches portant sur ce qu'il est coutume d'appeler «oral représenté » connaissent actuellement un regain d'intérêt, après quelques travaux fondateurs plus anciens (en particulier Cerquiglini 1981).

C'est à la notion d'oral représenté et à ses marques linguistiques que nous intéresserons ici. Nous nous appuierons pour cela sur les travaux de C. Marchello-Nizia (2012 et à par.) et sur la définition qu'elle propose de cette forme particulière d'écrit qui se donne comme de l'oral (« discours rapporté se donnant comme l'exacte reproduction de paroles ayant été prononcées oralement », $2012: 247$ )

Plusieurs travaux de recherche récents ont permis de révéler certaines des spécificités linguistiques qui semblent distinguer assez nettement les séquences d'oral représenté du reste des textes au Moyen Âge (Guillot \& al 2013 et à par., Glikman \& Mazziotta 2013). Un ensemble de recherches complémentaires a porté sur la signalisation du discours rapporté (Marnette 2006a et b) et sur la délimitation linguistique et graphique de l'oral représenté dans les textes et les manuscrits médiévaux (voir notamment Cerquiglini 1981, Llamas-Pombo 2010, Marchello-Nizia 2012).

La recherche que nous présentons ici a été menée dans cette double perspective : centrée sur l'incise, notre étude porte sur l'une des marques linguistiques qui semble dès le départ servir au typage et au repérage d'unités discursives de différentes natures: placée tantôt au début d'une séquence d'oral représenté qui se démarque du récit et qui est constituée d'un ou de plusieurs tours de parole, tantôt à l'intérieur des sous-segments correspondant aux tours de parole qui se succèdent à l'intérieur de la séquence, l'incise a sans nul doute une fonction organisationnelle. Les travaux de C. Marchello-Nizia ont également déjà montré que, dans sa forme-même, l'incise partage, avec d'autres marques organisationnelles (annonce et rappel, cf. infra), certains des traits qui caractérisent fortement la grammaire de l'oral représenté au Moyen Âge : présence d'un verbum dicendi, sur-représentation du présent de l'indicatif, fréquence de l'expression du sujet, nature sémantique du sujet (humain), fréquence de l'ordre $\mathrm{VS}^{2}$.

Ce sont ces différents contextes d'occurrence et les propriétés formelles et fonctionnelles de l'incise qui seront analysés dans la suite de cet article, dans une perspective diachronique et contrastive, notre corpus rassemblant des textes en vers et en prose et relevant de différents domaines discursifs. En ce sens, notre étude prolongera et complètera pour la période postérieure les recherches déjà développées par C. Marchello-Nizia sur les plus anciens textes français (voir notamment Marchello-Nizia à par.). 


\section{Méthodologie, corpus et problématique}

\subsection{Méthodologie et corpus}

La présente étude s'inscrit dans une série de travaux sur l'oral représenté réalisés à partir du corpus de textes de la Base de français médiéval (http://txm.bfm-corpus.org) (Guillot \& al. 2013 et à par.). A la différence des travaux précédents où nous avions privilégié des méthodes quantitatives (analyse de spécificités et analyse factorielle des correspondances) sur des ensembles de textes relativement importants, nous développons à présent à une analyse qualitative fine d'occurrences annotées manuellement, ce qui nous oblige à restreindre fortement les dimensions du corpus traité.

Le corpus analysé est composé de six textes désignés ci-après par leur sigle (s'il existe) dans la liste de référence du Dictionnaire étymologique de l'ancien français $^{3}$ (voir les références complètes ci-dessous). Il s'agit de deux romans en vers de la seconde moitié du $12^{\text {ème }}$ siècle (EneasS2 et YvainKu), d'une chronique en prose du début du $13^{\text {ème }}$ siècle (RobClariL), d'une chronique en prose de l'extrême fin du $14^{\text {ème }}$ s. (FroissChron3 $\mathrm{D}^{4}$ ), d'un roman en prose de la fin du $14^{\text {ème }}$ siècle (BerinB) et d'un ensemble de nouvelles du début du $15^{\text {ème }}$ siècle (QJoyesR). Malgré ses dimensions restreintes, le corpus est assez diversifié : sont représentés des textes d'ancien et de moyen français, en vers et en prose, littéraires et historiques. Tous les textes sélectionnés sont narratifs, puisque c'est dans la narration que l'oral représenté est le plus fréquent.

Dans les textes sélectionnés, nous avons recherché à l'aide du logiciel $\mathrm{TXM}^{5}$ les frontières du discours direct ${ }^{6}$ marquées par des guillemets dans les éditions de référence et balisées avec la balise TEI $<\mathrm{q}>^{7}$ dans la Base de français médiéval. Nous avons également recherché les changements de tour de parole dans les dialogues, qui sont le plus souvent repérables grâce à l'usage du tiret dans les éditions. Les requetes ont été adaptées aux pratiques éditoriales et à l'état du balisage propre à chaque texte. Par exemple, on utilise le requête « $<\mathrm{q}>[]$ » pour sélectionner les premiers mots du discours direct. Les résultats des requêtes ont été exportés sous forme de tableaux de concordances et annotés à l'aide du logiciel Microsoft Excel.

Nous avons relevé dans les six textes du corpus tous les contextes d'occurrence possibles de l'incise, les débuts de séquence d'oral représenté d'une part, les changements de tour de parole d'autre part. L'annotation des changements de tour de parole a été exhaustive dans tous les textes sauf dans BerinB et dans FroissChron3D, où nous avons analysé des échantillons de 40000 mots environ. Notre analyse s'est par ailleurs limitée aux 50 premières et 50 dernières occurrences des débuts de séquences d'oral représenté dans ces deux textes.

Les caractéristiques des textes annotés sont présentées dans le tableau ci-dessous :

\begin{tabular}{|c|c|c|c|c|}
\hline \multirow[t]{2}{*}{ Texte } & \multirow{2}{*}{$\begin{array}{c}\text { Date de } \\
\text { composition }\end{array}$} & \multicolumn{2}{|c|}{ Nombre de mots } & \multirow{2}{*}{$\begin{array}{l}\text { Nombre } \\
\text { d'incises }\end{array}$} \\
\hline & & total & $\mathrm{OR}^{8}$ & \\
\hline BerinB & ca. 1370 & $\begin{array}{r}39807 \\
\text { (sur 147799) } \\
\end{array}$ & 13624 & 89 \\
\hline EneasS2 & ca. 1155 & 34958 & 12896 & 34 \\
\hline FroissChron3D & vers 1400 & $\begin{array}{r}40274 \\
\text { (sur 217105) }\end{array}$ & 4710 & 68 \\
\hline QJoyesR & ca. 1400 & 34632 & 11313 & 216 \\
\hline RobClariD & après 1205 & 34013 & 3454 & 40 \\
\hline YvainKu & ca. $1177-1181$ & 41740 & 19463 & 123 \\
\hline
\end{tabular}

Tableau 1 : Dimensions du corpus

BerinB : Roman de Berinus, édité par R. Bossuat, Paris, SATF 1931.

EneasS2 : Eneas, édité par J. Salverda de Grave, Paris, Champion, 1925.

FroissChron3D : Jean Froissart, Chroniques, éditées par G. T. Diller, Genève, Droz, 1972.

QJoyesR : Quinze joies de mariage, éditées par J. Rychner, Genève, Droz, 1963. 
RobClariL Robert de Clari, Conquête de Constantinople, éditée par P. Lauer, Paris, Champion, 1924. YvainKu : Chrétien de Troyes, Chevalier au Lion ou Yvain, édité par P. Kunstmann, Ottawa ; Nancy, Université d'Ottawa, Laboratoire de Français Ancien ; ATILF, 2009.

\subsection{Problématique}

Comme on l'a indiqué plus haut, les incises se rencontrent toujours dans les situations de prise de parole, parmi lesquelles nous distinguerons deux cas.

L'incise peut d'une part marquer un premier tour de parole (désormais TP), éventuellement suivi d'autres TP. Ce premier TP peut être précédé d'une « annonce » (Marchello-Nizia 2012), c'est-à-dire d'un énoncé qui explicite par un verbum dicendi l'entrée dans une séquence d'oral représenté ${ }^{9}$ (désormais OR), configuration dans laquelle le locuteur moderne tend à voir une certaine redondance entre marques dont la forme et la fonction semblent très proches :

(1) Elle crolla lo chief chenu, / al Troïen a respondu. / « Sire», fait el, « voiz ci l'entree / del grant anfer la plus baee [...] »(EneasS2, v. 2294-2297)

L'incise peut d'autre part être associée à un ou plusieurs TP dans le cadre d'une succession de TP. Dans ce cas, on peut considérer qu'elle permet d'expliciter l'alternance des TP :

(2) Si amenoit avant ses enfans, tant que uns sages hons de le chité respondi : «Ouil», fist chis, «nous connissons bien que che fu femme Kyrsaac et que che furent si enfant. - Ba!» fist li marchis, " pour coi ne connissiés vous dont l'un des enfans a seigneur? Je le vous dirai », fist chis (RobClariL, p. 99)

Il a en effet été suggéré que la fonction première de l'incise n'est peut-être pas de lever une possible ambiguïté référentielle quant au référent locuteur du segment dans lequel elle se trouve, mais plutôt de servir de «borne » (Cerquiglini, 1981 : 31). Franzén, dans son étude sur les pronoms personnels, considère que le pronom sujet de l'incise n'a pas « la fonction de faire ressortir nettement quelle est la personne qui parle » (1939: 100), remarque qui s'appuie sur l'étude de la Conqueste de Constantinople de Robert de Clari, texte en prose du début du $13^{\text {ème }}$ siècle qui a pour caractéristique, nous le verrons, d'associer fréquemment annonces et incises, et dans lequel, par conséquent, le locuteur moderne tend à percevoir une certaine redondance dans l'expression répétée de la source à l'origine de l'énonciation.

Comme le souligne B. Cerquiglini, au Moyen Âge, le dispositif d'encadrement de l'oral représenté peut être assez différent dans le vers et dans la prose, la prose semblant favoriser la multiplication et la redondance des marques. Dans son étude contrastive de la Chanson de Roland et de la Queste del Saint Graal, C. Marchello-Nizia observe toutefois que les cas de double marquage (annonce + incise) sont rares dans ces deux textes, les incises étant davantage employées dans les TP internes à l'épisode d'OR. Ce constat semble contredire l'hypothèse de B. Cerquiglini, qui souligne au contraire que l'entrée dans l'OR exige un marquage plus important et plus explicite que les TP qui se succèdent ensuite : en effet, la parole est alors déjà là, et les échanges pourraient davantage se passer d'explicitation.

On verra que notre étude ne permet pas de valider pleinement ces différentes hypothèses : nous n'avons pas observé, pour l'ancien français, de différence très nette entre textes en vers et textes en prose et, par ailleurs, l'incise est fréquente en entrée de séquence d'OR dans le vers et se surajoute parfois à l'annonce. Nous proposerons une hypothèse diachronique pour rendre compte de ces variations.

Pour mener notre analyse, nous avons retenu différents critères de description. Pour ce qui est de la forme de l'incise, nous avons considéré la nature du sujet, toujours présent dans l'incise, le lexème verbal et le temps du verbe, et la présence éventuelle d'un objet. Nous avons par ailleurs déterminé la fréquence de l'incise, quand elle est associée au premier TP et dans le cadre d'alternances de TP. Nous avons observé plus précisément, d'une part les cas où il y a co-présence d'une annonce et d'une incise, d'autre part les cas sans incise dans le cadre d'une succession de TP. 


\section{Caractéristiques morpho-syntaxiques de l'incise}

Dans les six textes de notre corpus, les incises présentent des points communs et des différences. Elles contiennent ainsi toutes un verbum dicendi et un sujet exprimé, postverbal, et ne mentionnent jamais l'interlocuteur (on ne trouve pas li/lor dit/fait il). Par ailleurs, au sein d'un même texte, l'incise tend à avoir une structure assez régulière, qu'elle soit placée en début d'épisode d'OR ou à la frontière des TP.

\subsection{Yvain, Eneas et Clari}

Dans les deux textes en vers et rapprochés chronologiquement, Eneas et Yvain, l'incise présente une structure très proche, qu'elle suive un premier $\mathrm{TP}$ ou prenne place dans une succession de TP avec changement de locuteur.

Dans Eneas, l'incise prend presque toujours la même forme: sur les 34 occurrences, on rencontre 33 structures identiques : le verbe faire au présent avec un pronom personnel de $3^{\text {ème }}$ personne inversé, sans objet :

(3) De ce qu'il ot ot grant peor, / arestut soi, si escolta / et la prestresse araisona. / «Dame », fait il, « ce que puet estre ? [...]» (EneasS2, v. 2710- 2713)

Un seul exemple fait exception, au niveau du lexème verbal, de la nature du sujet et de l'expression de l'objet, sous forme d'un pronom démonstratif. L'incise est cependant au présent, comme dans les autres exemples :

(4) «[...] molt me dotai de cel ostage / que vos eüstes an Cartage, / n'an perdissiez tot vostre afaire / ou vos tornast a grant contraire. / - Peres », ce respont Eneas, / « ge ne pooie laissier pas /ne venisse parler a vos [...] » (EneasS2, v. 2853-2859)

Il est notable que le verbe de l'incise est au présent quel que soit le temps du verbe de parole introducteur de la séquence d'OR (s'il y en a un) ${ }^{10}$. Les exemples 3 (ci-dessus, araisona) et 5 , éloignés de 80 vers, sont remarquables de ce point de vue :

(5) [...] il s'abandone / vers la dame, se l'araisone. / «Dame», fait il, «por moie amor / avez sofert mortel dolor [...]» (EneasS2, v. 2626-2632).

Dans Yvain, 118 des 123 incises contiennent pareillement le verbe faire, et seulement 5 le verbe dire. L'incise est très majoritairement au présent (on ne rencontre que 2 occurrences de dire au passé, l'une au passé simple, l'autre à imparfait) :

(6) Et a sa dame a conseillié / Que revenuz est ses messages, / Si a esploitié come sages. / « Comant? fet ele. Quant venra / Messire Yveins ? » (YvainKu, v.18961900)

Comme dans Eneas, une incise au présent est compatible avec un verbum dicendi précédent à un temps passé (mais les exemples sont plus rares).

Le sujet, toujours exprimé, prend majoritairement la forme d'un pronom personnel, mais de manière bien moins écrasante que dans Eneas (les occurrences de l'incise sont certes plus nombreuses). On trouve ainsi le pronom personnel dans 77 cas (63\%). Plus précisément, le pronom personnel apparaît comme la forme nettement privilégiée dans les premiers TP (21 occurrences sur 25 , les 4 autres étant des sujets nominaux ou des noms propres). Il l'est en revanche beaucoup moins dans les successions de TP : 56 occurrences sur les 95 incises (57\%), alors qu'on trouve par ailleurs 35 syntagmes nominaux et noms propres, 6 pronoms démonstratifs et un indéfini. Le verbe et le sujet sont très majoritairement au singulier : on ne rencontre que 2 occurrences de font $\mathrm{il}$, et une occurrence dans laquelle on observe une disjonction d'accord entre le sujet (2 pronoms démonstratifs conjoints) et le verbe :

(7) Mes bien sache li rois, mes sire, / Que je sui de ceste bataille / Oltrez et recreanz sanz faille. /- Mes ge.- Mes ge ", fet cil et cil. / Tant sont andui franc et gentil / Que la victoire et la querone / Li uns a l'autre otroie et done; (YvainKu, v.63486354) 
Comme dans Eneas, la présence d'un objet est rarissime : on n'en trouve qu'une seule occurrence, qui comporte le verbe dire à l'imparfait :

(8) «Comant! Seroiz vos or de çax, / Ce disoit messire Gauvains, / Qui por leur fames valent mains? » (YvainKu, v.2485-2488)

Ecrasante dans Eneas, la structure fet-il/fet-ele, est majoritaire aussi dans Yvain (57\%).

Dans les deux textes l'incise peut se combiner avec d'autres éléments linguistiques, reconnus comme des marques de début de discours direct (Cerquiglini 1981, Marchello-Nizia 2012, Diachroniques 2013), en particulier les termes d'adresse, les interjections, les modalisateurs énonciatifs (par exemple certes), mais aussi les verbes à l'impératif, les interrogatifs directs.

Dans La Conqueste de Constaninople de Robert de Clari (désormais Clari), texte légèrement postérieur à Eneas et Yvain, en prose et relevant du domaine historique, les caractéristiques morpho-syntaxiques des incises se démarquent en partie de celles des deux textes précédents. En effet, sur les 40 incises, le verbe faire est utilisé dans 38 cas mais il est au passé simple dans 34 cas $^{11}$ :

(9) $[\ldots]$ s'asanlerent un jor et pourparlerent une grant traïson $[\ldots]$; tant que Morchofles dist : "Se vous me voliés », fist il, "croire, et vous me voliés faire empereur, je vous deliverroie si bien des Franchois et de l'empereur que jamais warde n'en ariés. » (RobClariL, p.61)

Les deux seules occurrences de verbe au présent ont un sujet non humain (et il s'agit en outre, plus que de contexte d'oral, de « lecture » d'un écrit sur un support) :

(10) et li autres ymages tendoit main en un vilain lieu, si disoit : «Ichi» fait li images, « les boutera on. » Ches deux ymages si seoient devant le cange qui molt soloit estre rikes illuec [...] (RobClariL, p. 88)

Hormis le verbe faire, on trouve une occurrence du verbe commenchier et une de dire, toutes deux au passé simple. Le verbe faire domine donc, comme dans Eneas et Yvain, mais il est presque toujours au passé simple.

Pour ce qui est du sujet, Clari présente des caractéristiques en partie différentes de celles des deux textes précédents. Ainsi, le sujet ne prend la forme d'un pronom personnel que dans 13 des 40 cas : on trouve 3 occurrences de pronom démonstratif, et, surtout, 24 cas de sujet nominal :

(11) Aprés quant li marchis fu croissiés, si dist as barons : «Seigneur, » fist li marchis, «ou vaurrés vous passer, ne en quel tere de Sarrasins vaurés vous aller? » (RobClariL, p. 6)

De plus, et ce point rapproche Clari d'Yvain, le pronom personnel n'est pas systématiquement au singulier : sur les 14 occurrences, on en trouve 3 au pluriel.

On observe donc des différences notables entre ces trois textes d'ancien français, mais, au sein d'un même texte, les structures sont en petit nombre.

\subsection{Quinze joies de mariage, Berinus et Froissart}

Les incises présentes dans les trois textes de moyen français peuvent sembler formellement assez hétérogènes. Les deux textes qui présentent des similarités génériques ou formelles (récits longs), Berinus (vers 1370) et les Chroniques de Froissart (vers 1400), désormais Froissart, partagent des caractéristiques communes qui les démarquent assez nettement des textes de la période antérieure. Les incises des Quinze joies de mariage, désormais Quinze joies, (1400), texte qui s'apparente à un recueil de nouvelles plutôt qu'à un récit long, ressemblent davantage à celles des textes d'ancien français ${ }^{12}$. Nous commencerons notre présentation par ce texte.

Dans les Quinze joies, les incises sont particulièrement fréquentes ${ }^{13}$ (216 occurrences). Malgré leur nombre bien plus élevé que dans les autres textes, elles présentent des caractéristiques morpho- 
syntaxiques très régulières. Les verbes faire (195 occurrences) et dire (21 occurrences) se partagent les occurrences. Comme dans Eneas et Yvain, le verbe est toujours au présent (hormis 2 occurrences de dire au passé simple). Il est cependant vrai que, à l'inverse de ces deux textes, les verbes des contextes narratifs qui précèdent sont le plus souvent au présent.

Le sujet est majoritairement représenté par le pronom personnel (159 occurrences, 74\%) et plus rarement par un syntagme nominal (57 occurrences, 26\%). Comme dans les textes les plus anciens du corpus, les pronoms personnels dominent donc, mais la part des syntagmes nominaux (Sn) n'est pas négligeable. On ne relève pas de pronom démonstratif.

L'exemple suivant associe les deux types de sujet:

(12) - Ha a, belle dame, fait il, c'est mal dit.- Avoy, monseigneur, dit la nourrice, vous ne savés pas la paine que madame y a et qu'ilz nous font endurer a les nourrir. - Par ma foy, dit la chambriere, monseigneur, c'est grant honte a vous quant vous venez dehors [...] (QJoyesR, p. 31)

Dans Berinus, nous avons relevé 89 incises ${ }^{14}$. Elles comprennent toutes le verbe dire, généralement sous la forme dist. On peut hésiter sur le statut temporel de cette forme, qui peut s'interpréter comme un présent ou un passé simple (voir Marchello-Nizia 2012 : 254). Mais l'entourage textuel de la forme dist comporte beaucoup plus souvent des verbes au passé simple que des verbes au présent, ce qui l'oppose à la forme dit, qui présente la caractéristique inverse (et qui apparaît sporadiquement dans les incises aussi) :

(13) haulça la paulme et en donna au varlet une grant buffe, et puis lui dist (BerinB, p. 17)

(14) car il dit bien ce que dire doit (BerinB, p. 176)

Il nous paraît donc préférable d'analyser dist comme une forme de passé simple dans ce texte.

Les sujets syntaxiques ne prennent que très exceptionnellement la forme du pronom de $3^{\text {ème }}$ personne $(3$ occurrences sur un total de 89$)$. Le nom propre $(\mathrm{Np})$ domine (55 occurrences, $62 \%)$, mais le $\mathrm{Sn}$ est très fréquent aussi (31 occurrences, 35\%). Enfin, quelques-unes des incises de ce texte se distinguent par le fait qu'elles contiennent un pronom objet (17 occurrences de 'ce dist $\mathrm{Np} / \mathrm{Sn}$ ') :

(15) « Ma doulce mere, ce dist Aigres, Dieu fera de moy sa voulenté [...] » (BerinB, p.377).

Le texte de Froissart contient un total de 68 incises ${ }^{15}$. Ce texte se différencie de tous les autres par son usage massif du verbe respondre (50 occurrences), les verbes dire (17 occurrences) et demander (1 occurrence) étant nettement moins fréquents. Comme dans le texte précédent, les incises sont au passé simple (la forme respondi n'est pas ambiguë) et le sujet est le plus souvent nominal (44 Sn, 65\% et 13 $\mathrm{Np}, 19 \%)$. Le pronom de $3^{\text {ème }}$ personne est assez rare (11 occurrences, $\left.16 \%\right)$, même s'il est moins exceptionnel que dans Berinus. La forme canonique de l'incise est 'respondi Sn' (36 occurrences, 53\%).

L'étude contrastive des incises dans ces six textes permet d'établir des regroupements qui peuvent correspondre à une évolution diachronique de la forme des incises. Eneas/Yvain et Berinus/Froissart se placent aux deux extrémités du spectre : prévalence du verbe faire, du présent de l'indicatif et du pronom personnel pour les premiers, prévalence d'autres verba dicendi (dire pour Berinus, respondre pour Froissart), verbe au passé simple, et diminution très nette de l'usage du pronom de $3^{\text {ème }}$ personne pour les seconds. Le texte de Clari et les Quinze joies présentent des caractéristiques mixtes mais utilisent, comme les quatre autres textes, un nombre très réduit de structures.

\section{Fréquence et caractéristiques des incises selon les contextes}

Nous étudierons la fréquence et la fonction des incises dans chacune de deux configurations définies en 2.2. Nous mettrons ainsi en évidence, sur l'ensemble de la période considérée, l'évolution de la 
distribution des incises en entrée dans la séquence d'OR et en situation de changement de locuteur. L'ancien et le moyen français présentent de ce point de vue des configurations très différentes.

Voici ci-dessous une vue synthétique de la distribution des incises dans les différents textes. Le tableau montre un renversement de tendance dans l'usage de l'incise, d'abord relativement fréquente en entrée dans la séquence d'OR, puis massivement utilisée pour les changements de locuteur :

\begin{tabular}{|l|c|c|c|}
\hline & $\begin{array}{c}\text { Incise Entrée } \\
\text { OR }\end{array}$ & $\begin{array}{c}\text { Incise Changement } \\
\text { locuteur }\end{array}$ & Total \\
\hline Eneas & 31 & 3 & 34 \\
\hline Yvain & 25 & 98 & 123 \\
\hline Clari & 28 & 12 & 40 \\
\hline Quinze Joies & 26 & 190 & 216 \\
\hline Berinus & 11 & 78 & 89 \\
\hline Froissart & 5 & 63 & 68 \\
\hline
\end{tabular}

Tableau2 : Distribution des incises en entrée dans l'OR et en situation de changement de locuteur

\subsection{TP en première position et entrée dans le discours}

Lorsque le TP occupe la première position (qu'il soit suivi ou non d'autres TP), nous avons distingué deux cas : le référent du locuteur du TP est le même que le sujet de la proposition qui précède, ou bien au contraire il en diffère. Dans le premier cas, le référent-locuteur est déjà saillant, dans le second il ne l'est pas. On parlera dans le premier cas de continuité référentielle et dans le second de discontinuité référentielle (nous avons par ailleurs pris en compte le fait que la proposition qui précède soit une annonce ou non, comme on le verra plus bas).

Indépendamment de la distinction entre ces deux situations, on peut faire l'hypothèse de deux fonctions possibles pour les incises dans cette position : baliser l'entrée dans le discours direct et/ou indiquer qui parle, fonction pour laquelle la distinction entre continuité et discontinuité référentielle devrait être pertinente. Signalons d'ores et déjà que, quand l'incise se trouve à l'intérieur du dialogue, ces mêmes fonctions se confondent plus ou moins dans la mesure où l'alternance des TP est constitutive du dialogue : les marques de l'alternance permettent à la fois de structurer l'épisode d'oral représenté en différents TP et d'identifier le locuteur du segment-hôte. On notera que l'incise, pour ces deux fonctions, apparaît fréquemment avec d'autres marques, en particulier les termes d'adresse, comme en témoignent plusieurs de nos exemples (voir les exemples $3,4,11,12$ et 15 ci-dessus).

\subsubsection{Yvain, Eneas et Clari}

Bien que les incises présentes dans les deux textes en vers de notre corpus, qui sont également les textes les plus anciens, soient formellement assez proches, leurs contextes d'usage sont globalement assez différents. Dans Eneas, les incises sont très majoritairement utilisées au moment de l'entrée dans la séquence d'oral représenté ; dans Yvain, elles sont moins fréquentes dans cette position. Comme annoncé plus haut, nous nous limitons dans cette section à l'analyse des incises placées en début de séquence et nous intéressons plus particulièrement aux contextes dans lesquels elles marquent soit la continuité, soit la discontinuité référentielle.

Dans Eneas, sur les 69 cas d'entrée dans le discours ( $\left.1^{\mathrm{er}} \mathrm{TP}\right)$, la très grande majorité (68 cas) correspond à une situation de continuité référentielle. Dans ce contexte, l'incise est relativement fréquente (30 occurrences, $44 \%$ ). 
Si l'on considère l'expression du sujet dans la proposition qui précède immédiatement le TP (qu'elle annonce explicitement celui-ci ou non), on constate que le sujet est exprimé dans $71 \%$ des occurrences (48 sur 68), sous forme d'un sujet nominal, d'un nom propre ou d'un pronom personnel. De plus, dans la majorité des 20 cas où le sujet n'est pas exprimé, le verbe est coordonné à un autre verbe dont le sujet est explicite (ou plus rarement, précédé d'une subordonnée dont le sujet est explicite) :

(16) Elle crolla lo chief chenu, / al Trö̈en a respondu. / «Sire», fait el, « voiz ci l'entree / del grant anfer la plus baee [...] » (EneasS2, v. 2295-2298)

Dans de rares énoncés, la mention explicite du référent sujet est relativement éloignée :

(17) Li escuiers lo salua, / a une part traist lo marchis,/ au chief d'un dois se sont asis, / son mesage li prent a dire. / «Antant a moi », fait il, « biaus sire; / la raïne m'a ça tramis / et mande toi qu'en cest païs / est novelment uns hom venuz / de çaus de Troie, des veincuz. (EneasS2, v. 3406-3414)

Dans ce dernier type d'occurrences, on ne peut exclure l'hypothèse que l'incise joue un rôle d'explicitation référentielle ; mais, d'une part le nombre de cas reste marginal, et d'autre part il arrive que l'incise, dans un cas analogue, soit absente :

(18) Ele lo huche et aceine, / amors l'argüe et demoine, /ne la laira, ce m'est avis, / desi qu'ele ait un trebuch pris. / Quant voit que ses amis s'en vait / et que s'amor a mort la trait, / si recomence a sospirer,/ a soi meïsme a dementer : / « Heu lasse, vait s'en il issi / que faz ge donc que ne m'oci ? (EneasS2, v. 1967-1976)

Dans la seule occurrence de discontinuité référentielle, la phrase qui précède peut être considérée comme porteuse d'une ambiguitté quant à la détermination du référent le plus saillant ; toutefois, indépendamment de l'incise, présente, l'utilisation du vocatif permet d'identifier le locuteur :

(19) Il li demande que ele a ; / se requerre lo velt de rien / que faire puisse, el l'avra bien / «Sire », fait ele, « que feras ? / Ge voi que mes fiz Eneas / est arivez an Lonbardie / o asez po de compaignie [...] » (EneasS2, v. 4308-4314)

Si l'on considère le verbe de la proposition qui précède le TP, dans les cas avec incise, on constate qu'il s'agit le plus souvent d'un verbum dicendi (dire, parler, araisoner...) et que la proposition sert donc à annoncer la séquence d'OR qui suit :

(20) Eneas est en piez levez, / de joie et de leece plore, / trestouz les dieus del ciel aore, / sa compaignie resbaudist ; / il nel sorent, tres qu'il lor dist. / « Seigneur, fait il, «c'est le contree / que nous avons tant desirree [...] » (EneasS2, v. 30643070)

Dans seulement 6 cas sur 30, le verbe précédent n'introduit pas le TP :

(21) Dido se pasme et color mue, / et quant sa mort a porveüe / « Anna », fait el, « or ai trové / molt buen consoil et esgardé [...] » (EneasS2, v. 1903-1906)

Dans la majorité des premiers TP, la présence de l'incise conduit donc à un double marquage de l'entrée dans l'épisode d'oral représenté (et à une sur-explicitation du locuteur). Ce qui rend l'interprétation difficile est le fait que les premiers TP sans incise sont précédés de propositions qui présentent les mêmes caractéristiques que celles précédant des TP avec incise (tant du point de vue de la mention du sujet que du type de verbe). Sans doute ne faut-il pas chercher ici une règle absolue, mais considérer l'incise « redondante » comme une option possible, au même titre que d'autres marques (interjections, termes d'adresse...) non systématiquement présentes.

La situation est assez différente dans Yvain, les incises étant moins abondantes en début de séquence, en particulier dans les cas de continuité référentielle. Ceux-ci représentent 138 des 145 cas d'entrée dans le discours $\left(1^{\text {er }} \mathrm{TP}\right)$, les situations de discontinuité référentielle étant donc, comme dans Eneas, très minoritaires (7/145 occurrences). On trouve une incise d'entrée dans 18 cas de continuité, soit seulement 13\% des occurrences (bien moins que dans Eneas), et dans les 7 cas de discontinuité référentielle. 
Sur la totalité des 21 pronoms présents dans les incises, 18 se trouvent donc dans les contextes de continuité référentielle, et 3 dans ceux de discontinuité référentielle, dans lesquels on rencontre aussi 4 sujets nominaux ou noms propres :

(22) Trestotes, a bien pres, le font, / Que de lor folie s'ancusent / Et ce qu'eles voelent refusent. / «Fui! fet ele, lesse m'an pes. / Se je t'an oi parler ja mes, / Ja mar feras, mes que t'an fuies [...] » (YvainKu, v.1646-1651)

(23) Lors veïssiez genz arriers treire ; / Trestoz li plus hardiz s'an fuit. / «Estez, fet messire Yvains, tuit. / Por coi fuiez ? » (YvainKu, v.6454-6457)

Dans ces cas avec incise, le verbe de la proposition qui précède le TP est un verbum dicendi à 10 reprises; il ne s'agit jamais de dire, mais d'autres verbes assez variés (appeler, repondre, refuser, comancer, comancer a pleidoier, metre a reison, redire, saluer, etc.) :

(24) Maintenant qu'il fu relevez / De pasmeisons, si l'apela : / « Dex! fet ele, que voi ge la ? / Qui est qui se demante si ?» (YvainKu, v.3562-3565)

Dans les entrées sans incise, le verbe dire est au contraire largement dominant (même si on relève quelques occurrences de repondre, crier, etc.). Autrement dit, la forme de l'annonce est un peu différente selon qu'elle se combine avec l'incise (le verbe dire est exclu) ou qu'elle est employée seule (le verbe dire domine largement).

Dans quelques cas (4), l'incise indique le changement de locuteur après une séquence de discours indirect :

(25) Et a sa dame a conseillié / Que revenuz est ses messages, / Si a esploitié come sages. / «Comant ? fet ele. Quant venra / Messire Yveins ?» (YvainKu, v.1896-1900)

Mais il arrive presqu'aussi souvent (3 occurrences), que l'incise suive une séquence de discours indirect sans qu'il y ait discontinuité référentielle.

(26) Et li rois dit qu'il partira / A bien et a foi la querele. / « Ou est, fet il, la dameisele / Qui sa seror a fors botee / De sa terre et deseritee / Par force et par male merci ? [...]» (YvainKu, v.63766381)

Dans Yvain, l'incise, le plus souvent réalisée avec un pronom personnel, ne marque donc qu'assez rarement l'entrée dans le discours direct (25/145 cas), sauf dans les situations de discontinuité référentielle, elles-mêmes minoritaires.

Clari se trouve dans une position intermédiaire par rapport aux deux textes précédents. Sur les 91 cas d'entrée dans le discours $\left(1^{\text {er }} \mathrm{TP}\right)$, on rencontre une incise dans 28 cas $(30 \%$, c'est-à-dire moins que dans Eneas, 46\%, mais plus que dans Yvain, 17\%).

Plus précisément, sur les 86 cas de continuité référentielle (qui constituent donc la majorité des occurrences), on a une incise dans 23 cas (soit $27 \%$, contre $46 \%$ dans Eneas et $13 \%$ dans Yvain). L'incise est par ailleurs présente dans les 5 cas de discontinuité référentielle. Rappelons que l'incise a pour sujet un $\mathrm{Sn}$ dans 14 cas (dont les 5 cas de discontinuité référentielle), un pronom personnel dans 13 cas, et un pronom démonstratif dans 1 cas.

Si l'on considère tout d'abord les cas de discontinuité référentielle, on constate que, dans 4 cas, le TP est précédé d'une séquence de discours indirect :

(27) Quant il furent revenu et il se furent desarmé, si merchia molt durement li empereres le marchis de chou qu'il l'avoit si bien vengié de chu sien anemi, tant que li marchis li demanda pour coi il avoit fait les portes fremer aprés lui : «Ba! ensi est ore! » fist li empereres. (RobClariL, p. 34)

Dans l'un des cas, le référent du locuteur est totalement absent de la proposition qui précède :

(28) Si comme il le recouvroit, si s'aparut Nostre Sires a lui, si parla a lui ; or avoit li boins hons une toaile entour lui : «Cha donne», fist Nostre Sire, « chele 
toaile », et li boins hons li bailla, et Nostre Sires en envolepa sen visage

(RobClariL, p. 83)

Dans ces 5 cas, on pourrait interpréter l'incise comme un moyen d'expliciter le référent locuteur, non saillant dans le contexte précédent.

L'examen des contextes de continuité référentielle où l'incise est présente (23 cas sur 86 ) montre que le référent est explicité dans la proposition qui précède dans $11 \mathrm{cas}$, le plus souvent sous forme nominale (7 occurrences de sujet nominal préverbal et 3 occurrences de sujet postverbal), et sous forme d'un pronom personnel dans un seul cas :

(29) Quant li empereres le vit venir, si se leva encontre lui et s'en fist molt grant feste. Si demanda li empereres as barons : «Savés vous ore », fist il, " qui chist hons est? - Sire, nennil », fisent li baron. (RobClariL, p. 55)

Quand le sujet n'est pas explicité dans la proposition qui précède immédiatement le TP (12 cas), le verbe sans sujet est juxtaposé ou coordonné à une proposition dans laquelle le référent est explicité (sous forme nominale), ou bien il suit une subordonnée dans laquelle il l'est pareillement :

(30) Adont furent trestout li baron de l'ost mandei et li Venicien, et quant il furent tot assanlé, si se leva li dux de Venice, si parla a aus : « Seigneur » fist li dux, " or avons nous raisnauvle acoison d'aler en Coustantinoble, se vous le loés; » (RobClariL, p.32)

Dans un seul cas, la mention explicite du référent sujet est très éloignée.

On trouve les mêmes possibilités quant à la nature du référent dans la proposition qui précède le TP pour les 13 cas où l'incise comporte un pronom personnel : le sujet peut être exprimé sous forme nominale, ou ne pas être exprimé. Il est donc difficile de déterminer ce qui motive en incise un sujet nominal ou pronominal. Il est pareillement malaisé de justifier l'occurrence unique de pronom démonstratif :

(31) $\mathrm{Si}$ amenoit avant ses enfans, tant que uns sages hons de le chité respondi: "Ouil », fist chis, "nous connissons bien que che fu femme Kyrsaac et que che furent si enfant [...]» (RobClariL, p.99)

Considérons maintenant le verbe de la proposition qui précède le TP. Dans les 5 cas de discontinuité référentielle, la proposition qui précède correspond majoritairement (mais il ne s'agit que de 4 occurrences) à une séquence de discours indirect, à laquelle le TP réagit :

(32) Et chil de dedens respondirent qu'il n'i enterroient, tant que li marchis vint as murs, si dist qu'il n'i enterroit : «Ba! comment? » fist le rois, « de ne sui jou sires et rois de laiens? (RobClariL, p. 36)

Dans les 23 cas de continuité référentielle avec incise, on trouve toujours un verbum dicendi, majoritairement dire (13 occurrences), mais aussi respondre, parler et demander, souvent précédés de l'adverbe si (on ne rencontre qu'à une seule reprise une séquence de discours indirect dans la proposition précédente) :

(33) Si comme il furent la venu, si leur dist li dux : « Seigneur, » fist il, « nous avons pris consel entre mi et me gent en tele maniere que [...]» (RobClariL, p11)

Comme dans Eneas, la présence d'un double marquage, tant du référent-locuteur que de l'entrée dans le discours (annonce et incise), n'est pas rare dans Clari: on trouve cette configuration dans presqu'un tiers des cas. Ce que le locuteur moderne tend à considérer comme un effet de redondance est particulièrement remarquable quand le référent est explicité par deux syntagmes nominaux successifs. Comme dans Eneas, l'interprétation de la présence de l'incise est rendue difficile par le fait que les premiers TP sans incise sont précédés de propositions qui présentent les mêmes caractéristiques (tant en ce qui concerne la mention du sujet dans la proposition précédente que le type de verbe).

Nous présentons dans le tableau ci-dessous un récapitulatif de la fréquence des incises dans les cas de continuité et de discontinuité référentielle dans les 3 textes d'ancien français du corpus : 


\begin{tabular}{|l|c|c|c|c|}
\hline & \multicolumn{2}{|c|}{ Continuité référentielle } & \multicolumn{2}{c|}{ Discontinuité référentielle } \\
\hline & Présence incise & Absence incise & Présence incise & Absence incise \\
\hline Eneas & $30-44 \%$ & $38-56 \%$ & 1 & 0 \\
\hline Yvain & $18-13 \%$ & $120-87 \%$ & 7 & 0 \\
\hline Clari & $23-27 \%$ & $63-73 \%$ & 5 & 0 \\
\hline
\end{tabular}

Tableau 3 : fréquence des incises en entrée dans l'OR

\subsubsection{Quinze joies de mariage, Berinus et Froissart}

Deux évolutions majeures semblent se dessiner à la fin du Moyen Âge : d'une part l'incise devient de plus en plus rare en tout début de séquences d'OR et elle se spécialise dans le marquage de l'alternance des locuteurs à l'intérieur de la séquence dialoguée; d'autre part le pronom personnel de troisième personne devient marginal (cf. 3.2). De ce point de vue, la répartition en deux sous-ensembles, avec les Quinze joies d'un côté et Berinus/Froissart de l'autre, semble se confirmer, comme on va le voir.

Dans les Quinze joies, l'incise se rencontre dans 26 des 94 cas d'entrée dans le discours direct $(28 \%$ d'entrées avec incise, comme dans Clari). Dans 79 énoncés, le locuteur du $1^{\text {er }}$ TP correspond au sujet de la proposition qui précède (continuité référentielle), et, parmi eux, 12 présentent une incise (18\% : moins que dans Clari et Eneas, mais davantage que dans Yvain). Comme dans les textes précédents, les cas de discontinuité référentielle sont relativement rares (15 énoncés) et l'incise est à peu près systématique dans ce contexte (14 occurrences).

Comme on l'a vu plus haut (3.2), le sujet est majoritairement pronominal dans les incises (4 Sn et 22 pronoms personnels). Mais il est surtout présent en cas de discontinuité référentielle (dans les 14 incises marquant une discontinuité référentielle, on trouve 11 pronoms personnels et $3 \mathrm{Sn}$ ). On ne peut totalement exclure que l'incise contribue dans ce cas à expliciter le référent-locuteur (comme le fait également le vocatif) :

(34) et le bon home auxi vient de la ville ou d'ailleurs de ses besongnes et lui demande que elle a. «Sire, fait elle, je suy corrocee, car l'enfant est trop malade (lequel, en effect, est tout sain). Il est, fait elle, si chault que c'est merveille et m'a dit la nourrice qu'il a deux jours [...]» (QJoyesR, p. 67)

Dans cet exemple, le référent-locuteur du TP est en effet présent dans l'annonce sans être pour autant l'élément le plus saillant (le sujet est enchâssé dans un complétive). Dans d'autres cas, le locuteur du TP est totalement absent de l'annonce.

L'absence d'incise est donc très rare dans les contextes potentiellement ambigus (1 seule occurrence) :

(35) Lors le proudomme, qui est vaincu, demande qui les menera et quelles femmes yront en leur compaignie. «Par ma foy, mon compere ou mon cousin, il y vient vostre dame la mere de ma cousine vostre femme et la femme de tel et de tel, et son cousin et le vostre, et les autres femmes de nostre rue ou d'environ nous [...]» ( QJoyesR, p.14)

Elle est présente en particulier lorsque le discours direct fait suite à un énoncé au discours indirect :

(36) Lors s'en va Jouhanne et trouve le gallant, qui ly demande quelles nouvelles de sa dame. «Par Dieu, fait elle, je la trouve a recommencer, mais, pour ce que m'en suy meslee, je vouldroye bien que vous fussés a ung, car j'ay paour que elle me descouvre a son mary ou a ses amis. » (QJoyesR, p. 46)

Parmi les rares occurrences (12) de l'incise en contexte de continuité référentielle, celle-ci ne prend qu'une seule fois la forme d'un $\mathrm{Sn}$ (on trouve en revanche 11 pronoms personnels). 
(37) Et quant elle s'est ung pou esbatue et voit la bonne voulenté de son mary, elle a aucuns de ses amis qui traictent avecques la mere, qu'elle die qu'elle a tourjours esté avecques elle et que la pouvre fille s'en estoit allee pour ce qu'il la vouloit affoller. « Je ameroy mieulx, fait la mere au mary, que vous la me baillassez du tout que la batre ainxin [...]» (QJoyesR, p . 80)

A l'inverse de Clari, ce texte ne présente pas de double marquage du Sn.

On note par ailleurs que l'incise n'est précédée d'une annonce que dans 8 de ces 12 cas (verbe dire ou demander). Dans les 67 énoncés sans incise qui marquent également la continuité référentielle, le verbum dicendi introducteur du discours direct est toujours présent. Il s'agit majoritairement du verbe dire.

Dans Les Quinze Joies, l'emploi de l'incise en début de séquence d'OR semble coïncider majoritairement avec des situations dans lesquelles l'entrée dans la séquence est annoncée moins explicitement.

Dans Berinus, la proportion d'incise en entrée de séquence est très faible (11 incises sur 100 occurrences, $11 \%$ ) et l'incise semble être exceptionnelle en cas de continuité référentielle : sur 91 cas, elle n'est présente qu'à 2 reprises.

L'incise continue en revanche à s'employer lorsque l'entrée dans le discours direct se double d'une discontinuité référentielle : dans les 9 cas concernés, l'incise est toujours présente. Elle suit un discours indirect dans 7 de ces 9 énoncés. L'incise indique alors une alternance des tours de parole (le début de la séquence au discours direct correspond en réalité à une première alternance de locuteurs) :

(38) et l'autre bourgois, qui estoit demourés avec Berinus, le mist a raison et lui demanda dont il estoit et comment il avoit nom et de quelx gens il estoit estraiz : «Sire », dit-il, "l'en m'appelle Berinus et fu né a Romme [...]» (BerinB, p. 41)

Dans les 2 autres cas, l'incise ne suit pas un discours indirect mais elle marque également le changement de l'agent :

(39) Dont apella Gieffroy la bourgoise et elle y vint : «Dame », dist il, « ce poise moi que vostre nepveu s'est fait congnoistre a vous ; maiz puis qu'il est ainsi, dame, je vous pry que vous soiez soingneuse du celler, car vous savez le meschief qui en puet avenir »(BerinB, p. 365)

On a vu plus haut que les cas où l'incise n'indique pas l'alternance du locuteur mais la continuité référentielle sont très marginaux (2 occurrences). On note alors l'absence d'un verbum dicendi à l'intérieur de l'énoncé qui précède le discours direct :

(40) et pour ce lui convint il dire par force, car quelque chose que on lui deïst, feust de sa mere ou de sa suer, si le deïst a l'empereur, et le deüst on pour ce pendre ou ardoir. « Sire », dist Marcus, « puis qu'il vous plaist, il est droit que je vous die verité, quoy qu'il en doie avenir. » (BerinB, p.129)

Il s'agit là en réalité d'un trait qui caractérise plus généralement, dans ce texte, les incises d'entrée. Et il semble qu'il y ait une corrélation très forte entre l'absence d'annonce et la présence de l'incise : l'absence d'annonce conduit toujours à l'usage de l'incise, à deux exceptions près. Dans les 2 cas, le locuteur du discours direct correspond au sujet syntaxique de la proposition qui précède :

(41) Et quant le roy entendi qu'il n'avroit nulle certaineté du fait, si fu plus courroucié que devant : «Helas ! on souloit dire et voir estoit que Romme estoit la fontaine de toute la science du monde et en couroit le renom par tous païs, mais quant on savra la nouvelle de ceste aventure, qui moult tost sera espandue par plusieurs terres, chascun dira que $[\ldots] »^{16}($ BerinB, p. 411$)$

Le pronom personnel n'est employé que dans 3 de ces incises d'entrée. Il marque 2 fois le changement de locuteur, une fois la continuité référentielle.

Les grandes tendances qui caractérisent le texte de Berinus semblent se confirmer dans le texte de Froissart. L'incise y est très rare en entrée dans la séquence d'OR. Elle est quasiment impossible en cas 
de continuité référentielle (1 seule occurrence sur 96 cas de continuité) mais elle s'emploie assez systématiquement lorsque le locuteur n'est pas le sujet de l'énoncé qui précède (4 incises dans les 4 cas de changement référentiel).

4 des 5 incises d'entrée indiquent ainsi de manière claire le changement de locuteur. Dans 3 cas, le discours direct fait suite à un discours indirect:

(42) La fu pris li congiés, et baissa la roine a son departir tous les enfans, l'un apriés l'autre, de Hainnau, et aussi fist son fil Edouwars. Phelippe de Hainnau, qui puis fu roine d'Engleterre, conmença trop fort a plorer, qant li jones Edouwars prist congiet. On li demanda pourquoi elle ploroit : " Pour ce, dist elle, que mon biau cousin Edouwars d'Engleterre se depart de moi, et je l'avoie ja apris. » (FroissChron3D, p.70)

Dans le dernier cas, l'incise ne suit pas un discours indirect :

(43) messires Hues li Espensiers avoit ja acquis tant d'amis par ses dons et presens, lesquels il avoit donnés et la envoiiés et envoioit encores tous les jours, que toute dissimulation estoit en place et portoient trop fort partie a l'encontre de la roine d'Engleterre. «Et quel cose porons nous faire? » respondi adont la roine. « Dame », dist li contes de Qent [...] (FroissChron3D, p.56)

Le seul cas où l'emploi de l'incise n'est pas concomittant avec le changement de locuteur est également le seul cas où l'incise est précédée d'un verbum dicendi qui annonce le discours direct :

(44) Puis appella li rois Ainmeri de Pavie et li dist a part : « Je voel, dist li rois, que tu poursieves ton marchiet. Gautiers de Manni retournera avoecques toi, et de tout ce que il te conselle, uses apriés son consel. » Il respondi et dist: ... (FroissChron3D, p.863)

Il est donc exceptionnel que l'incise d'entrée se surajoute à l'annonce de la séquence d'OR. Et, comme dans le texte précédent, on observe que l'absence d'annonce favorise l'usage de l'incise. Le seul exemple qui ne suit pas cette tendance est un passage au discours direct qui fait suite à une séquence de discours indirect et qui présente une rupture de construction :

(45) et que elle fesist tant que elle euist .CCC. ou .CCCC. armeures de fier, car li langages dou prononchier pour le temps de lors estoit tels, et les amenast ou pais, et li Londriien les deliveroient de tous poins et se meteroient en lor compagnie : «Car par ma foi, messire Jehan et biau cousins, je n'ai de quoi faire ce paiement. Je n'ai finance fors que pour mes menus frés. » (FroissChron3D, p.61)

On note que, dans ce cas aussi, le locuteur de la séquence de discours direct correspond au sujet de la proposition qui précède.

Les 2 seules occurrences du pronom personnel dans les incises d'entrée marquent le changement de locuteur après du discours indirect.

Comme pour les textes d'ancien français, nous présentons ci-dessous, pour les textes de moyen français, un récapitulatif de la fréquence des incises dans les cas de continuité et de discontinuité référentielle:

\begin{tabular}{|l|l|l|r|r|}
\hline & \multicolumn{2}{|c|}{ Continuité référentielle } & \multicolumn{2}{c|}{ Discontinuité référentielle } \\
\hline & Présence incise & Absence incise & Présence incise & Absence incise \\
\hline Quinze Joies & $12-15 \%$ & $67-85 \%$ & 14 & 1 \\
\hline Berinus & $2-2.2 \%$ & $89-97.8 \%$ & 9 & 0 \\
\hline Froissart & $1-1 \%$ & $95-99 \%$ & 4 & 0 \\
\hline
\end{tabular}

Tableau 4 : Fréquence des incises en entrée dans l'OR 


\subsection{Enchaînements de TP et changements de locuteurs}

\subsubsection{Eneas, Yvain et Clari}

Dans Eneas, contrairement aux entrées dans l'OR (premier TP), les incises dans les enchaînements de TP sont très rares. Ainsi sur les 50 TP concernés, seulement 3 (soit 6\%) ont une incise, ce qui est très peu comparé, d'une part aux deux autres textes d'ancien français, et d'autre part à la fréquence de l'incise en $1^{\text {er }}$ TP. Sur les 3 occurrences, 2 se trouvent dans le dernier TP d'un échange de nombreux TP brefs (15 TP et $8 \mathrm{TP})$. On a 2 cas de 'fait pronom personnel' et 1 cas de 'ce respond $\mathrm{Sn}$ ' :

(46) « [...] el ne m'a mie deceü. / Fiz, molt avez sofert peors, / poines, travalz et granz dolors, / molt me dotai de cel ostage / que vos eüstes an Cartage, / n'an perdissiez tot vostre afaire / ou vos tornast a grant contraire. / - Peres », ce respont Eneas, / « ge ne pooie laissier pas / ne venisse parler a vos [...]» (EneasS2, v. 2850-2859)

(47) - Ja fetes vos voz nes garnir. / - Gié ? - Voire, volez moi foïr. / - Ainz m'an irai tot a veüe. / - Por coi m'avez donc deceüe ? / Deguerpiroiz me vos ansi ? / - Ge ne puis mes remaindre ci. / - Por coi ? fait el. - Li deu n'ont cure. / - Ohi lasse, quel aventure ! / Por coi ne sui ge donc ocise ? / Tant mar ai fet lo bel servise, / lo bel sanblant, lo bel ostage, / que ge vos ai fet en Cartage [...]» (EneasS2, v. 1679-1690)

C'est d'autant plus étonnant que l'on rencontre l'incise dans $44 \%$ des cas d'entrée dans l'OR, et ce alors qu'il s'agit très majoritairement de cas de continuité référentielle (le seul cas de discontinuité référentielle présente aussi une incise). Dans ce texte l'incise semble donc servir à baliser l'entrée dans l'OR plus que l'alternance des TP. Cette hypothèse est corroborée par le fait que le pronom personnel est très fréquent dans les incises : c'est une forme potentiellement ambiguë, mais cela importe peu si l'incise ne sert pas principalement à identifier le locuteur.

L'observation des successions de TP laisse paraître quelques échanges avec de nombreux TP sans aucune incise (15 TP, $8 \mathrm{TP}, 10 \mathrm{TP}$ et $13 \mathrm{TP})$ de longueur variable : dans 2 des échanges, les TP sont très brefs (moins de 10 mots) ; leur taille est plus fluctuante dans les autres (voir les exemples 46 et 47 ci-dessus).

Il arrive aussi que les échanges soient assez brefs ( 3 occurrences avec seulement 2 ou 3 TP). Enfin, on trouve quelques TP isolés (c'est-à-dire simplement précédés d'un premier TP, étudié en 2.1) sans incise, comme l'exemple suivant de 42 vers (que nous abrégeons) :

(48) molt ot grant duel, forment sospire, / des poinz se fiert, ses chevols tire. / « He las, chaitis, quel la ferai /de mon ami que perdu ai ? / Porté li ai molt male foi / quant il est pris ne morz sanz moi ; / [...] Retornerai la ou ge fui, / iluec ou ge parti de lui ;/se ge nel truis, po pris ma vie ; /se il n'est autres qui m'ocie, / sai ge bien que ge m'ocirrai, / soantre lui petit vivrai. » (EneasS2, v. 5142-5184)

Dans Yvain, les incises, qui sont assez rares au moment de l'entrée dans l'OR, sont au contraire relativement fréquentes lors des changements de TP. Ainsi sur les 206 TP concernés, 98 (soit 47.6\%) ont une incise, chiffre qui distingue Yvain d'Eneas, dans lequel les incises sont au contraire très rares dans ce contexte. L'incise reste cependant encore minoritaire.

L'incise présente dans ce contexte les mêmes caractéristiques que lorsqu'elle accompagne un premier TP, c'est-à-dire que le verbe faire au présent est largement dominant. De même, le pronom personnel est majoritaire, mais sa fréquence est cependant moindre que dans les incises d'entrée (56 occ. soit $57 \%$ des cas, contre $84 \%$ en incise d'entrée). On trouve par ailleurs des syntagmes nominaux ou des noms propres $(36 \%)$, des pronoms démonstratifs $(6 \%)$, et un pronom indéfini.

Pour ce qui est de la longueur des enchaînements de TP sans incise, on constate que les passages avec de nombreux TP sont rares. 
Dans Yvain, l'incise marque donc plus souvent le changement de locuteur dans le cadre des enchaînements de TP que l'entrée dans l'OR (sauf en cas de discontinuité référentielle). Sa forme est très proche dans les deux contextes, mais la fréquence des pronoms personnels, comparée à celle des sujets nominaux ou noms propres, est moins dominante dans les cas d'alternance de TP que dans les incises d'entrée (qui marquent en général une continuité référentielle).

Dans Clari, sur les 12 TP qui correspondent à un changement de locuteur, l'incise est systématiquement présente. Rappelons qu'elle l'est pareillement, en entrée dans l'OR, lorsqu'il y a discontinuité référentielle. L'incise apparaît donc systématiquement lorsque le locuteur n'est pas le référent le plus saillant, et qu'il y a donc risque d'ambiguïté référentielle.

(49) $[\ldots]$ tant que li marchis li demanda pour coi il avoit fait les portes fremer aprés lui : « $\mathrm{Ba}$ ! ensi est ore !» fist li empereres. «Or, de par Dieu !» fist li marchis. Et ne demora waires aprés que li empereres et si traïteur pourparlerent une grant traïson, que il voloit faire le marchis destruire. (RobClariL, p. 34)

Le sujet de l'incise est nominal dans 10 cas, c'est un pronom démonstratif dans 2 cas.

Dans l'un d'eux, le premier TP de l'échange comporte aussi une incise dont le sujet est un pronom démonstratif (c'est d'ailleurs la seule occurrence de démonstratif avec un premier TP). On notera que les deux démonstratifs désignent le même référent:

(50) Si amenoit avant ses enfans, tant que uns sages hons de le chité respondi : « Ouil », fist chis, « nous connissons bien que che fu femme Kyrsaac et que che furent si enfant. - Ba!» fist li marchis, «pour coi ne connissiés vous dont l'un des enfans a seigneur? Je le vous dirai », fist chis, «alés en Constantinoble et faites loi coroner; et quant il ara sis en le caiiere Coustentin et nous le sarons, adont si en ferons chou que faire en deverons. » (RobClariL, p. 99)

La configuration des successions de TP est très différente de celle observée dans Yvain: au plus, on trouve une succession de 3 TP (en comptant le TP initial), comme dans l'exemple donné ci-dessus.

\subsubsection{Quinze joies, Berinus et Froissart}

Sur les 248 TP qui correspondent à un changement de locuteur dans le texte des Quinze joies, l'incise est présente dans 190 cas (77\%), ce qui est légèrement moins fréquent que les cas d'entrée dans le discours avec discontinuité référentielle mais beaucoup plus que les cas d'entrée avec continuité). Ces incises contiennent $53 \mathrm{Sn}$ et 137 pronoms personnels (proportions très proches de celles de l'incise d'entrée avec discontinuité référentielle : 3/8). Sur les 53 cas de Sn on dénombre 18 occurrences de l'autre (deX)/l'une $(d e X)$.

Il est difficile de cerner ce qui justifie l'absence d'incise dans 58 cas. On observe parfois de microsystèmes, comme dans l'exemple donné ci-dessous (incise pour l'un des deux locuteurs, absence d'incise pour l'autre, mais apostrophe mon amy).

(51) El dit ainxin : «Pleust a Dieu, mon amy, que vous ne le feissés jamés si je ne vous en parlasse premierement ! - Et comment, fait il, ne le feriez vous point ? Par mon ame, mon amy, non, je cuide que nanil, et me semble que je en vauldroie mieulx. Et si j'en eusse autant sceu avant que je fusse mariee, je ne l'eusse esté jamés.- Quoy dea, fait il, et pourquoy vous mariastes vous doncques ?- Par ma foy, mon amy, je ne scey ; je estoie jeune fille et fasoie ce que mes pere et mere me disoient (combien que, a l'aventure, el en avoit bien tasté davant).- Qu'est ce a dire ? fait il. Je ne vous trouve nullefois sinon en ceste oppinion. Je ne scey que c'est.- Par mon ame, mon amy, si ce n'estoit vostre plaisir, je n'en vouldroye point. » (QJoyesR, p. 38)

Sur les 58 énoncés sans incises, l'apostrophe est présente dans 47 cas (81\%) et permet d'identifier clairement l'interlocuteur (ma dame, ma comère, m'amye, un $\mathrm{Np}$...) : 
(52) el lui dit : «Pour Dieu, fait el, lessés moy ester et actendez devers le matin. Certes, m'amie, non feroy; tournez vous devers moy. - Par Dieu, mon amy, vous me faites grant plesir si vous me lessés ester jusques a matin. » (QJoyesR, p. 36)

On note que, lorsque l'incise apparaît, la présence d'une apostrophe est bien moins fréquente (65 cas sur 190 soit 34\%). D'autres éléments initiaux peuvent s'employer (interjections, modalisateurs, etc.) ou pas. L'exemple suivant illustre les deux possiblilités :

(53) «[...] Mais face Dieu sa voulenté ! - Avoy, m'amie, fait le proudome, vous estes bien esmeue et sans cause.- Sans cause ? fait elle. Par Dieu, sans cause n'est ce pas ! [...] 》 (QJoyesR, p. 24)

Dans Berinus, l'incise est devenue habituelle (mais non obligatoire) en cas d'alternance des TP : elle est présente dans 78 des 90 changements de TP $(87 \%)$. La fréquence de l'incise dans ce contexte explique que la succession de plusieurs TP sans incise soit relativement rare. L'exemple le plus long du texte est le suivant (5 TP) :

(54) « [...] Ha ! Dieu, pere, pour Dieu mercy, dictes le moy et je le feray par Dieu. Beaux filz, je le vous diray moult voulentiers ; je vous pry tout avant que vous emportez de ce tresor a tel planté que jamaiz... voulez croire mon conseil, tost m'arez geté de paine. Pour Dieu, beaux filz, si vous hastez, car la nuit s'en va durement - - Ha ! Dieu, pere, pour Dieu mercy, dictes le moy et je le feray par Dieu - Beaux filz, je le vous diray moult voulentiers ; je vous pry tout avant que vous emportez de ce tresor a tel planté que jamaiz n'en aiez disete et le mettez en recoy bien en sauf; et quant vous revendrez, si me delivrerez de... avant que vous emportez de ce tresor a tel planté que jamaiz n'en aiez disete et le mettez en recoy bien en sauf ; et quant vous revendrez, si me delivrerez de ceste doulour ou je suis. Or vous hastez avant qu'il soit jour ". - Pere, ains vous osteray tout avant. - Par Dieu, beaux filz, jamaiz de cy je n'istray si avrez fait ce que je vous prie. » (BerinB, p. 395-396)

On note que chaque TP débute avec un terme d'adresse qui permet d'identifier le locuteur de chaque segment.

Dans le texte de Froissart, l'incise est également habituelle en cas d'alternance des TP (73 changements de locuteur, 63 incises, soit 86\%). Comme dans le cas précédent, il est rare que beaucoup de TP se succèdent sans incise. L'exemple le plus long de l'extrait analysé est le suivant :

(55) Se li recorda li chevaliers tout ensi conme il li estoit avenu. «Vous ne le poés amender, dist messires Jehans de Hainnau. Vous ont il mis a finance ? »«Oil»- «Et a conbien? »- «A douse cens florins, et m'ont demandé se vous demorriés pour moi. »- «Oil, dist il, et tantos se la sonme estoit plus grande diis fois. » (FroissChron3D, p.332)

Dans ces exemples, 3 tours de parole s'enchaînent; ils sont encadrés par une incise au début et à la fin de la séquence d'OR.

\section{Conclusion}

L'annotation d'un ensemble de six œuvres médiévales a permis l'analyse assez précise de la forme et de la fonction des incises utilisées dans ces textes. Elle en a souligné quelques propriétés permanentes, mais a révélé aussi d'importantes variations permettant de regrouper les textes : les deux œuvres en vers du $12^{\text {ème }}$ siècle, Eneas et Yvain, qui, malgré un usage différent de l'incise dans les situations d'alternance de $\mathrm{TP}$, présentent de grandes similitudes, de même qu'à l'autre extrémité temporelle les textes en prose de Berinus et de Froissart.

On peut faire l'hypothèse que ces variations reflètent l'évolution diachronique qu'a connue l'incise entre les $12^{\text {ème }}$ et $15^{\text {ème }}$ siècles. Peu spécialisée dans les premières œuvres, elle a d'abord une double fonction : elle participe au marquage de l'entrée dans l'épisode d'oral représenté ainsi qu'à la signalisation des 
changements de tours de parole. Elle se rapproche en cela d'autres marques linguistiques (termes d'adresse, exclamatifs, annonce, etc.) avec lesquelles elle se combine souvent. A la fin du Moyen Âge, la forme et la fonction de l'incise semblent s'être modifiées : spécialisée dans le marquage de l'alternance des locuteurs, elle se trouve rarement en début de séquence et ne se combine plus avec l'annonce. Sa forme s'est également transformée : le passé simple a succédé au présent et le pronom personnel sujet a cédé la place aux expressions nominales ( $\mathrm{Sn}$ et $\mathrm{Np}$ ) qui permettent une meilleure identification du référent locuteur.

Il faudrait bien entendu un corpus plus étendu que le nôtre pour confirmer ces points. Il est possible également que les usages de l'incise soient conditionnés aussi par d'autres facteurs que l'évolution diachronique. On a noté la situation particulière dans notre corpus des Quinze joies de mariage, légèrement postérieures à Berinus et à Froissart et, par certains côtés, formellement proches des plus anciens textes. Il se peut que le genre de la nouvelle, parce qu'il réunit un nombre de personnages très limité, souvent assimilés à des rôles sexués et opposés (par exemple le mari et la femme), favorise l'emploi du pronom personnel dans l'incise, à un moment où son sujet tend à être plutôt nominal dans les autres textes. Il est très probable par ailleurs que la structure métrique des textes en vers n'est pas sans lien avec la fréquence du balisage par l'incise : le début d'un tour de parole coïncide souvent avec le début d'un vers ou avec la césure, ce qui pourrait expliquer que les incises soient moins fréquentes en contexte d'alternance de TP dans le vers que dans la prose. Enfin, il est possible qu'à mesure que l'écrit se développe, son mode de conception et de diffusion s'éloigne de plus en plus de la modalité orale. Il est probable aussi que les textes en prose, et tout spécialement les récits historiques, se démarquent de cette modalité davantage que ne le font les romans en vers. Dans le contexte d'un récit destiné à une lecture orale, il n'est pas exclu que l'identification du locuteur puisse se faire (peut-être de façon assez mimétique) par d'autres facteurs que la mention explicite du nom, comme par exemple les gestes, l'intonation ... En revanche, dans un récit historique en prose, l'identification du locuteur par le texte pourrait être plus nécessaire ${ }^{17}$. Autant de pistes de recherche nouvelles à explorer à l'avenir...

\section{Références bibliographiques}

Cerquiglini, B. (1981). La parole médiévale : discours, syntaxe, texte. Paris : Editions de minuit.

Diachroniques (2013). Marques d'oralité en français médiéval, 3.

Franzén, T. (1939). Étude sur la syntaxe des pronoms personnels sujets en ancien français. Uppsala : Almqvist et Wiksells.

Gachet, F. (à par.) Incises de discours rapporté et autres verbes parenthétiques. Etude grammaticale. Paris: Champion.

Glikman, J. et Mazziotta, N. (2013). Représentation de l'oral et syntaxe dans la prose de la Queste del saint Graal (1225-1230). In : in Lagorgette, D. \& Larrivée, P. (éd.). Représentations du sens linguistique V, Chambery: Editions de l'Université de Savoie, 43-64.

Guillot, C., Lavrentiev, A., Pincemin, B. et Heiden, S. (2013). Oral représenté au Moyen Age : vers une définition et une méthodologie d'analyse. In : in Lagorgette, D. et Larrivée, P. (éd.). Actes du Colloque Représentations du sens linguistique V, Chambery : Editions de l'Université de Savoie, 17-41.

Guillot, C, Heiden, S. Lavrentiev, A., Pincemin, B. (à par.). L'oral représenté dans un corpus de français médiéval $\left(9^{\mathrm{e}}-15^{\mathrm{e}}\right)$ : approche contrastive et outillée de la variation diasystémique. Actes du colloque DIA, Copenhague : Université de Copenhague.

Llamas Pombo, E. (2010). Marques graphiques du discours rapporté (Manuscrits du Roman de la Rose, $\mathrm{XV}^{\mathrm{e}}$ siècle). In : Guillot C., Combettes B., Lavrentiev A., Oppermann-Marsaux E. \& Prévost S. (éd.). Le changement en français: Études de linguistique diachronique. Actes du colloque international DIACHRO-IV (22-24 octobre 2008, Madrid). Bern : P. Lang, 249-270.

Marchello-Nizia, Ch. (2012). L'oral représenté : un accès construit à une face cachée des langues 'mortes. In : Guillot C., Combettes B., Lavrentiev A., Oppermann-Marsaux E. \& Prévost S. (éd.). Le changement en français. Etudes de linguistique diachronique. Bern/Berlin/Bruxelles : Peter Lang, 247-264. 
Marchello-Nizia, C (à par.). Les débuts de l'« oral représenté » en français : marquage du discours direct dans les plus anciens textes. Mélanges Soutet.

Marnette, S. (2006a). La signalisation du discours rapporté en français médiéval. Langue française, 149, 31-47.

Marnette, S. (2006b). La ponctuation du discours rapporté dans quelques manuscrits de romans en prose médiévaux. Verbum, 1, 47-66.

\footnotetext{
${ }^{1}$ Le dernier numéro de la revue Diachroniques (2013), coordonné par A. Rodríguez-Somolinos dresse un état de ces recherches et propose des développements nouveaux.

${ }^{2}$ On notera que, sous la forme figée qu'on lui connaît (en français moderne et en français médiéval probablement aussi), l'incise n'est jamais utilisée dans le discours oral réel. Il s'agit donc d'un élément qui ne se rencontre qu'à l'écrit, mais dans le contexte spécifique de la représentation de l'oral. On peut en revanche trouver dans l'oral moderne des tournures liées au discours rapporté indirect: « ...qu'elle me dit » (voir Gachet à par.) ou direct ('oh là là j'ai dit') mais elles diffèrent assez nettement par leur forme des incises étudiées ici
}

${ }^{3} \mathrm{http}: / /$ www.deaf-page.de.

${ }^{4}$ Il s'agit de la troisième et dernière rédaction du premier livre des Chroniques de Froissart.

${ }^{5} \mathrm{http} / / /$ sourceforge.net/projects/txm

${ }^{6}$ Le discours direct (DD) fait partie de la séquence d'oral représenté, qui comprend en outre l'annonce, l'incise, et le rappel.

${ }^{7}$ http://www.tei-c.org/release/doc/tei-p5-doc/en/html/ref-q.html

${ }^{8}$ OR pour « oral représenté »; voir note 9 .

${ }^{9}$ L'annonce fait partie, comme l'incise et le rappel, des balises organisationnelles puisqu'elle ouvre la séquence d'oral représenté. Le rappel, dont il ne sera pas question dans ce travail, a pour fonction de clore le segment d'OR et de marquer le retour au récit.

${ }^{10}$ Les verbes de la proposition précédente sont d'ailleurs majoritairement au passé.

${ }^{11}$ Certes, dans ce texte, la très grande majorité des verbes de la narration précédant l'incise sont au passé simple ou à l'imparfait. Mais nous avons vu que, dans Eneas, alors que le verbe qui précède l'incise est le plus souvent aussi au passé, celle-ci est majoritairement au présent. Dans Yvain, on trouve avant l'incise, majoritairement au présent, à peu près autant de verbes au passé qu'au présent.

12 Il n'est pas certain que les raisons de cette hétérogénéité soient à rechercher dans les caractéristiques génériques des textes. Les trois textes d'ancien français inclus dans notre corpus étaient eux aussi des récits longs. Il faudrait un corpus plus étendu que le nôtre pour établir des liens entre la forme des incises et les types de textes.

${ }^{13}$ Le nombre de mots du texte n'est cependant pas plus élevé que celui des autres textes, et le nombre de mots des séquences d'OR est moins élevé que celui de Eneas, Yvain et Berinus. (voir tableau 1 dans 2.1.)

${ }^{14}$ Ce chiffre ne correspond pas au nombre total des incises utilisées dans Berinus, puisque nous n'avons dépouillé que 100 séquences d'entrée dans l'OR. Mais, dans ce texte, les incises sont très rarement employées en entrée de séquence d'OR (elles sont massivement utilisées pour marquer l'alternance des tours de parole). On peut donc supposer que la prise en compte de la totalité des séquences d'entrée ne modifierait pas beaucoup leur nombre total.

${ }^{15}$ Même remarque que pour le texte de Berinus (voir la note précédente).

${ }^{16}$ On note dans cet exemple le statut incertain de la première partie de ce qui a été interprété par l'éditeur comme du discours direct, mais qu'on aurait pu analyser comme du discours indirect libre (verbes à l'imparfait), ce qui conduit à rapprocher ce segment d'une forme d'annonce.

${ }^{17}$ Nous remercions l'un des évaluateurs d'avoir attiré notre attention sur ce dernier point. 